\title{
NEW AIRCRAFT CONFIGURATIONS HANDLING QUALITIES STUDIES BASED ON THE AERODYNAMIC INVARIANT CONCEPT
}

\section{J. Bazile}

AIRBUS Operations SAS

316 Route de Bayonne, Toulouse 3100, France

\begin{abstract}
This paper proposes an innovative approach to go deeper in the Handling Qualities knowledge for New Aircraft Configurations based on the Aerodynamic Invariant Concept. By manipulating the Doublet Lattice Method for aerodynamic derivatives computation, an Aerodynamic Invariant can be highlighted. It contributes to many aircraft dynamics parameters and can be considered as "key driver" of the longitudinal aircraft dynamics. The aim of this new approach is to study the impact of this aerodynamic invariant on aircraft Handling Qualities and to ask the question: "Could this aerodynamic invariant be used in parallel with Performance in the conceptual design phase to "seek" New Aircraft configurations capable of achieving further Handling Qualities improvement with Performance benefits?" The enclosed results highlight that the aerodynamic invariant could allow to improve and to optimize the aircraft Handling Qualities by relaxing the aircraft dynamics stability.
\end{abstract}

\section{NOMENCLATURE}

$\begin{array}{ll}\mathrm{Cm}_{q} & \text { Aircraft pitch damping derivative } \\ \mathrm{Cm}_{q}^{*} & \text { Aircraft pitch damping derivative at the neutral point } \\ \mathrm{Cm}_{\alpha} & \text { Aircraft stability static derivative } \\ \mathrm{Cm}_{\delta q} & \text { Elevator pitch effectiveness } \\ \mathrm{Cz}_{q} & \text { Aircraft lift due to pitch rate derivative } \\ \mathrm{Cz}_{\alpha} & \text { Aircraft lift curve slope } \\ \mathrm{Cz}_{\alpha}^{\text {htp }} & \text { Horizontal tailplane lift curve slope } \\ \mathrm{Cz}_{\alpha} \mathrm{Cm}_{q}^{*} & \text { Aerodynamic invariant } \\ \mathrm{Cz}_{\delta q} & \text { Elevator lift effectiveness } \\ \{\mathrm{Hc}\}_{z} & \text { Aircraft deformations in plunge at the control points with } \\ & \{\mathrm{Hc}\}_{z}=\{\cos \varphi\}\end{array}$




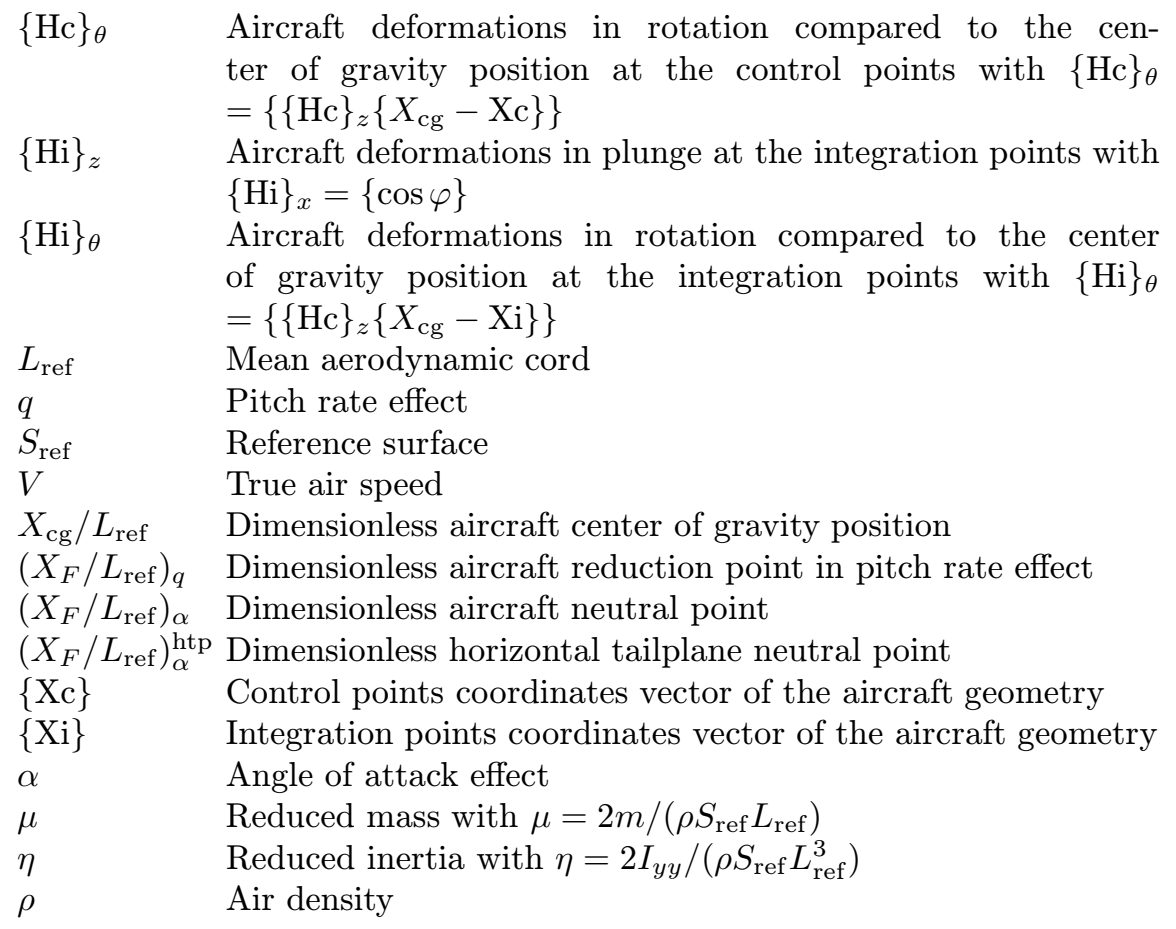

\section{INTRODUCTION}

On the topic of innovative aircraft configurations, this paper proposes a possible way allowing the identification and optimization of future aircraft concepts. Indeed, the Airbus A350 and the Boeing 787 represent "the best" of what aircraft manufacturers can do in term of Performance, mainly, and in terms of Handling Qualities with classical aircraft architecture. With these two optimized aircrafts, it seems that manufacturers are on an asymptotic curve in terms of Performance and Handling Qualities. Today, to increase the aircraft Performance and Handling Qualities, manufacturers should have to consider alternative ways to reach this aim. Based on this remark, the frame of the approach proposed is to consider the aircraft design, not only based on Performance criteria since the conceptual design phase but also, in parallel, based on Handling Qualities criteria.

By focusing on considerations linked to the Handling Qualities, this study aims at approaching this problem by exhibiting a parameter which will qualify Aerodynamic Invariant. It can be stated that this invariant is independent of the center of gravity position for a given Mach number. It contributes in many linear relations relative to aircraft dynamics, such as the short period mode frequency, 
damping ratio, and the transfer functions compared to elevator and wind. The analysis highlights that it is one of the main drivers of the longitudinal aircraft dynamics. The purpose of this paper is to study the impact of this aerodynamic invariant on aircraft Handling Qualities and to ask the main question: "Could this aerodynamic invariant be used in parallel with Performance in the conceptual design phase in order to "seek" New Aircraft Configurations capable of achieving further Handling Qualities improvement?" For example, is it possible to get less load factor in turbulence with the same level of elevator deflection or reach a given load factor with less elevator deflection? This aerodynamic invariant identified via the pitch damping derivative is negative on conventional aircraft configurations, but if it becomes less negative or zero, what is its effect on the aircraft dynamics in open or in closed loop and what is the aircraft geometry? Is it a relevant parameter in order to relax the aircraft dynamic stability?

This document is composed of three sections. Section 2 describes the aerodynamic invariant identification starting from the results coming from the Lifting Surface Theory [1]. After this rather theoretical first part, section 3 measures the aerodynamic invariant impact on the aircraft dynamics in closed loop with time simulations results. Furthermore, in order to approach this complex problem in a simple way, the time simulations are based on the short period mode model. The Performance aspect related to the drag equation will be treated in future works. Lastly, section 4 proposes to conclude this analysis and to trace the aerodynamic invariant outlook.

\section{LONGITUDINAL AERODYNAMIC INVARIANT IDENTIFICATION AND LINK WITH NEW AIRCRAFT CONFIGURATIONS}

The main object of this part concerns the identification of a parameter called the Aerodynamic Invariant. Subection 2.1 presents the two initial thoughts justifying the aerodynamic invariant identification. Subection 2.2 proposes to provide an answer to the first thought based on considerations resulting from the Doublet-Lattice Method [1]. Based on this result, subsection 2.3 highlights the aerodynamic invariant and subsection 2.4 authorizes an answer to the second thought. Subsection 2.5 proposes the interpretation of the aerodynamic invariant. Subsection 2.6 presents the closed link between the aerodynamic invariant and the short period mode model characteristic parameters such as the short period mode frequency, the damping ratio, and the dynamic stability limit point. Finally, subsection 2.7 proposes a discussion around the perspectives linked to the aerodynamic invariant identification based on the aircraft Handling Qualities point of view. 


\subsection{Initial Thoughts Leading to the Aerodynamic Invariant Identification}

This subsection presents the two initial thoughts leading to the Aerodynamic Invariant Concept.

\section{First thought}

Most of the time, to build aerodynamic derivatives transportation at a given center of gravity position, except for the lift curve slope $\mathrm{Cz}_{\alpha}$ and the elevator effectiveness $\mathrm{Cz}_{\delta q}$, it is necessary to identify their value at a reference point. This value is mainly defined at $25 \%$ of the mean aerodynamic cord.

To illustrate this remark, let focus on the pitch damping derivative, the most difficult to model with accuracy. So, the pitch damping derivative can be expressed at an $X_{M}$ position, noted $\mathrm{Cm}_{q}\left(X_{M}\right)$, according to the following expression including the $\mathrm{Cm}_{q}, \mathrm{Cz}_{q}$, and $\mathrm{Cm}_{\alpha}$ derivatives calculated at the 25 percent point, noted $X_{25}$. This formal expression is:

$$
\begin{aligned}
\mathrm{Cm}_{q}\left(X_{M}\right)=\mathrm{Cm}_{q}\left(X_{25}\right)+\left(\frac{X_{M}}{L_{\mathrm{ref}}}-\frac{X_{25}}{L_{\mathrm{ref}}}\right)\left(\mathrm{Cz}_{q}\left(X_{25}\right)-\mathrm{Cm}_{\alpha}\left(X_{25}\right)\right) \\
-\mathrm{Cz}_{\alpha}\left(\frac{X_{M}}{L_{\mathrm{ref}}}-\frac{X_{25}}{L_{\mathrm{ref}}}\right)^{2} .
\end{aligned}
$$

Here, the terms $\mathrm{Cz}_{q}\left(X_{25}\right)$ and $\mathrm{Cm}_{\alpha}\left(X_{25}\right)$ are easily identifiable by considering the classical formal expression of the static stability and lift due to pitch rate derivatives.

Furthermore, to calculate $\operatorname{Cm}_{q}\left(X_{M}\right)$, it is also necessary to know the pitch damping derivatives value $\mathrm{Cm}_{q}\left(X_{25}\right)$. This one is mainly provided by wind tunnel tests. Now, if there is no provided wind tunnel test data, one cannot calculate $\mathrm{Cm}_{q}\left(X_{M}\right)$ and the question addressed is: "How to identify the $\mathrm{Cm}_{q}\left(X_{25}\right)$ value based on an analytical expression?" Nevertheless, to calculate $\mathrm{Cm}_{q}\left(X_{M}\right)$, an empirical pitch damping derivative expression may be highlighted mainly based on the horizontal tailplane contribution, this formal expression being

$$
\mathrm{Cm}_{q}\left(X_{M}\right)=\mathrm{Cz}_{\alpha}^{\mathrm{htp}}\left(\frac{X_{M}}{L_{\mathrm{ref}}}-\left(\frac{X_{F}}{L_{\mathrm{ref}}}\right)_{\alpha}^{\mathrm{htp}}\right)^{2} .
$$

This expression frees from the knowledge of the pitch damping derivative at $25 \%$, but is only usable for classical aircraft configuration of type wing/fuselage/ horizontal tailplane. That means it is no more available for a flying wing. So, this remark involves the first question: "Can we exhibit an analytical pitch damping derivative expression without having to recourse to the reference point knowledge, whatever the aircraft configuration?" 


\section{Second thought}

This second thought was born from the analysis of the current manoeuvre point expression in stationary pull-up power-off resulting of the stability criteria in a manoeuvre. Indeed, the manoeuvre point formulation in European formalism is:

$$
\frac{X_{M P}}{L_{\mathrm{ref}}}=\left(\frac{X_{F}}{L_{\mathrm{ref}}}\right)_{\alpha}-\frac{\mathrm{Cm}_{q}\left(X_{\mathrm{cg}}\right)}{\mu} .
$$

By considering its definition, the manoeuvre point location is strongly independent of the center of gravity position. However, to calculate its position with exactness, the analytical formula (2) contains a parameter actually depending on the center of gravity position: the term $\mathrm{Cm}_{q}\left(X_{\mathrm{cg}}\right)$. This term necessarily involves the dependence of the manoeuvre point position compared to the center of gravity and, consequently, becomes incompatible with the above basic definition. So, an inconsistency is noticed. Then, a second question can be addressed as follows: "Can we exhibit an analytical maneouvre point expression independent of the center of gravity position?"

Here, the two starting thoughts are given in brief that justified the aerodynamic invariant identification. With time, these two thoughts have been converted into a real problem, in particular, for the manoeuvre point position, because simulations did not fit with those predicted by formula (2). Based on these two questions, the following parts bring answers.

\subsection{Answer to the First Question}

To answer the first question, the Doublet-Lattice Method [1] initially used for the unsteady aerodynamic calculation in the frame of Oscillating Lifting Surface Theory will be considered. However, by restricting this method in the stationary field, this one becomes identical to the Vortex Lattice Method [2]. Without going into calculation details, the fundamental longitudinal aerodynamic derivatives formulated in the aircraft frame, such as $\mathrm{Cz}_{\alpha}, \mathrm{Cz}_{q}, \mathrm{Cm}_{\alpha}$, and $\mathrm{Cm}_{q}$, can be expressed according to the following relations [3]:

$$
\begin{aligned}
\mathrm{Cz}_{\alpha}=\frac{\{\mathrm{Hi}\}_{z}^{t}\left[A_{0}\right]\{\mathrm{Hc}\}_{z}}{S_{\mathrm{ref}} L_{\mathrm{ref}}} ; & \mathrm{Cz}_{q}=\frac{\{\mathrm{Hi}\}_{z}^{t}\left[A_{0}\right]\{\mathrm{Hc}\}_{\theta}}{S_{\mathrm{ref}} L_{\mathrm{ref}}} ; \\
\mathrm{Cm}_{\alpha}=\frac{\{\mathrm{Hi}\}_{\theta}^{t}\left[A_{0}\right]\{\mathrm{Hc}\}_{z}}{S_{\mathrm{ref}} L_{\mathrm{ref}}} ; & \mathrm{Cm}_{q}=\frac{\{\mathrm{Hi}\}_{\theta}^{t}\left[A_{0}\right]\{\mathrm{Hc}\}_{\theta}}{S_{\mathrm{ref}} L_{\mathrm{ref}}} .
\end{aligned}
$$

By replacing $\{\mathrm{Hi}\}_{z},\{\mathrm{Hc}\}_{z},\{\mathrm{Hi}\}_{\theta}$, and $\{\mathrm{Hc}\}_{\theta}$ in the above terms, one obtains:

- the formal classical expression of static stability derivative, noted $\mathrm{Cm}_{\alpha}\left(X_{\mathrm{cg}}\right)$, as

$$
\mathrm{Cm}_{\alpha}\left(X_{\mathrm{cg}}\right)=\mathrm{Cz}_{\alpha}\left(\frac{X_{\mathrm{cg}}}{L_{\mathrm{ref}}}-\left(\frac{X_{F}}{L_{\mathrm{ref}}}\right)_{\alpha}\right) ;
$$


- the formal classical expression of the lift due to pitch rate derivative, noted $\mathrm{Cz}_{q}\left(X_{\mathrm{cg}}\right)$, as

$$
\mathrm{Cz}_{q}\left(X_{\mathrm{cg}}\right)=-\mathrm{Cz}_{\alpha}\left(\frac{X_{\mathrm{cg}}}{L_{\mathrm{ref}}}-\left(\frac{X_{F}}{L_{\mathrm{ref}}}\right)_{q}\right)
$$

- the novel pitch damping derivative expression, noted $\mathrm{Cm}_{q}\left(X_{\mathrm{cg}}\right)$, as

$$
\begin{aligned}
& \operatorname{Cm}_{q}\left(X_{\mathrm{cg}}\right) \\
= & -\mathrm{Cz}_{\alpha}\left(\left(\frac{X_{\mathrm{cg}}}{L_{\mathrm{ref}}}\right)^{2}-\frac{X_{\mathrm{cg}}}{L_{\mathrm{ref}}}\left(\left(\frac{X_{F}}{L_{\mathrm{ref}}}\right)_{\alpha}+\left(\frac{X_{F}}{L_{\mathrm{ref}}}\right)_{q}\right)+\left(\frac{X_{F}}{L_{\mathrm{ref}}}\right)_{\alpha, q}^{2}\right),
\end{aligned}
$$

with the point noted $\left(X_{F} / L_{\mathrm{ref}}\right)_{\alpha, q}^{2}$ defined as a "new" aerodynamic point. The "new" aspect lies in the fact that the flight mechanics and aerodynamics literature do not make mention of it and the associated aerodynamic properties are unknown.

Now, by the identification of expression (5), it is possible to answer the first question addressed in subsection 2.1: "It is possible to exhibit an analytical pitch damping derivative expression without having recourse to the reference point knowledge whatever the aircraft configuration."

Moreover, one notices that expression (5), not mentioned in flight mechanics literature, does not make any assumption on the geometrical aircraft configuration. It means that this expression can be used for conventional configuration of type wing/fuselage/tailplane as well as flying wing or others.

Furthermore, the determination of the pitch damping derivative at an unspecified point does not require any more knowledge of this one at a reference point, i. e., $25 \%$ of the mean aerodynamic cord. However, this novel pitch damping derivative expression can raise a question about the confidence on it.

It is difficult to bring a final answer; nevertheless, it can be said, first, that this method based on the lifting surface theory leads to the well known aerodynamic derivatives expressions such as $\mathrm{Cm}_{\alpha}\left(X_{\mathrm{cg}}\right)$ and $\mathrm{Cz}_{q}\left(X_{\mathrm{cg}}\right)$, widely mentioned in the flight mechanics literature. Second, starting from (5), one can demonstrate that the expression giving the pitch damping derivative at an $X_{M}$ point compared to the center of gravity $X_{\mathrm{cg}}$ is identical to that one met in flight mechanic handbooks and at the start of the first thought (1). These considerations authorize a good confidence in the highlighted pitch damping expression (5).

Consequently, based on the pitch damping derivative expression previously established, it is possible to highlight the Aerodynamic Invariant expression. 


\subsection{Aerodynamic Invariant Identification}

To identify the aerodynamic invariant, let consider the pitch damping derivative expression (5) and proceed as follows.

Looking at the quadratic equation (5), one notices the sum of the neutral point and the reduction point in pitch rate effect. This remark is not without reminding the property linked to the "remarkable identity," which can be expressed with the sum and the product of the roots, $\lambda_{1}$ and $\lambda_{2}$, so:

$$
I=\left(X-\lambda_{1}\right)\left(X-\lambda_{2}\right)
$$

and

$$
I=X^{2}-\left(\lambda_{1}+\lambda_{2}\right) X+\lambda_{1} \lambda_{2}
$$

Now, this result can be transposed to the pitch damping derivative expression. In order to highlight this property, it is necessary to add and subtract the product between the neutral point and the reduction point in pitch rate effect; so, the following relation is obtained:

$$
\begin{aligned}
& \mathrm{Cm}_{q}\left(X_{\mathrm{cg}}\right)=- \mathrm{Cz}_{\alpha}\left(\left(\frac{X_{\mathrm{cg}}}{L_{\mathrm{ref}}}\right)^{2}-\frac{X_{\mathrm{cg}}}{L_{\mathrm{ref}}}\left\{\left(\frac{X_{F}}{L_{\mathrm{ref}}}\right)_{\alpha}+\left(\frac{X_{F}}{L_{\mathrm{ref}}}\right)_{q}\right\}\right. \\
&\left.+\left(\frac{X_{F}}{L_{\mathrm{ref}}}\right)_{\alpha}\left(\frac{X_{F}}{L_{\mathrm{ref}}}\right)_{q}-\left(\frac{X_{F}}{L_{\mathrm{ref}}}\right)_{\alpha}\left(\frac{X_{F}}{L_{\mathrm{ref}}}\right)_{q}+\left(\frac{X_{F}}{L_{\mathrm{ref}}}\right)_{\alpha, q}^{2}\right)
\end{aligned}
$$

Then, by distributing the lift curve slope $C z_{\alpha}$ in a judicious manner, the following expression is obtained:

$$
\begin{array}{r}
\operatorname{Cm}_{q}\left(X_{\mathrm{cg}}\right) \\
=\underbrace{-\mathrm{Cz}_{\alpha}\left(\left(\frac{X_{\mathrm{cg}}}{L_{\mathrm{ref}}}\right)^{2}-\frac{X_{\mathrm{cg}}}{L_{\mathrm{ref}}}\left\{\left(\frac{X_{F}}{L_{\mathrm{ref}}}\right)_{\alpha}+\left(\frac{X_{F}}{L_{\mathrm{ref}}}\right)_{q}\right\}+\left(\frac{X_{F}}{L_{\mathrm{ref}}}\right)_{\alpha}\left(\frac{X_{F}}{L_{\mathrm{ref}}}\right){ }_{q}\right)}_{\text {Dependent on center of gravity }} \\
-\underbrace{\mathrm{Cz}_{\alpha}\left(\left(\frac{X_{F}}{L_{\mathrm{ref}}}\right)_{\alpha, q}^{2}-\left(\frac{X_{F}}{L_{\mathrm{ref}}}\right)_{\alpha}\left(\frac{X_{F}}{L_{\mathrm{ref}}}\right)_{q}\right)}_{\text {Independent of center of gravity }}
\end{array}
$$

Consequently, the expression (6) can be separated in two terms: the first term dependent on the center on gravity position and the second term independent of the center of gravity position, because only function of the aerodynamic forces reduction points as $\left(X_{F} / L_{\mathrm{ref}}\right)_{\alpha},\left(X_{F} / L_{\mathrm{ref}}\right)_{q}$ and $\left(X_{F} / L_{\mathrm{ref}}\right)_{\alpha, q}^{2}$. Then, by looking more closely, the first term is structured like the so-called "remarkable identity." Consequently, by introducing its reduced form in (6), this one becomes: 


$$
\begin{aligned}
\mathrm{Cm}_{q}\left(X_{\mathrm{cg}}\right)=-\mathrm{Cz}_{\alpha}\left(\frac{X_{\mathrm{cg}}}{L_{\mathrm{ref}}}-\right. & \left.\left(\frac{X_{F}}{L_{\mathrm{ref}}}\right)_{\alpha}\right)\left(\frac{X_{\mathrm{cg}}}{L_{\mathrm{ref}}}-\left(\frac{X_{F}}{L_{\mathrm{ref}}}\right)_{q}\right) \\
& -\mathrm{Cz}_{\alpha}\left(\left(\frac{X_{F}}{L_{\mathrm{ref}}}\right)_{\alpha, q}^{2}-\left(\frac{X_{F}}{L_{\mathrm{ref}}}\right)_{\alpha}\left(\frac{X_{F}}{L_{\mathrm{ref}}}\right)_{q}\right) .
\end{aligned}
$$

Now, observing (7), onr notices that the terms in the part dependent on the center of gravity position are not without reminding the expression of the static stability derivative $\mathrm{Cm}_{\alpha}\left(X_{\mathrm{cg}}\right)$ (3) and the lift due to pitch rate derivative $\mathrm{Cz}_{q}\left(X_{\mathrm{cg}}\right)$ (4) devised by the aircraft lift curve slope $\mathrm{Cz}_{\alpha}$. Consequently, by substituting (3) and (4) into (7), one obtains the following expression:

$$
\begin{aligned}
\mathrm{Cm}_{q}\left(X_{\mathrm{cg}}\right)=\frac{\mathrm{Cm}_{\alpha}\left(X_{\mathrm{cg}}\right)}{\mathrm{Cz}_{\alpha}} \mathrm{Cz}_{q}\left(X_{\mathrm{cg}}\right) & \\
& -\mathrm{Cz}_{\alpha}\left(\left(\frac{X_{F}}{L_{\mathrm{ref}}}\right)_{\alpha, q}^{2}-\left(\frac{X_{F}}{L_{\mathrm{ref}}}\right)_{\alpha}\left(\frac{X_{F}}{L_{\mathrm{ref}}}\right)_{q}\right) .
\end{aligned}
$$

Now, by defining the term independent of the center of gravity as $\mathrm{Cm}_{q}^{*}$, one obtains:

$$
\mathrm{Cm}_{q}^{*}=-\mathrm{Cz}_{\alpha}\left(\left(\frac{X_{F}}{L_{\mathrm{ref}}}\right)_{\alpha, q}^{2}-\left(\frac{X_{F}}{L_{\mathrm{ref}}}\right)_{\alpha}\left(\frac{X_{F}}{L_{\mathrm{ref}}}\right)_{q}\right) .
$$

By putting it into expression (8), one obtains:

$$
\mathrm{Cm}_{q}\left(X_{\mathrm{cg}}\right)=\frac{\mathrm{Cm}_{\alpha}\left(X_{\mathrm{cg}}\right)}{\mathrm{Cz}_{\alpha}} \mathrm{Cz}_{q}\left(X_{\mathrm{cg}}\right)+\mathrm{Cm}_{q}^{*} .
$$

Finally, by multiplying (10) by $\mathrm{Cz}_{\alpha}$, one achieves this analytical calculation with the following expression:

$$
\mathrm{Cz}_{\alpha} \mathrm{Cm}_{q}\left(X_{\mathrm{cg}}\right)-\mathrm{Cm}_{\alpha}\left(X_{\mathrm{cg}}\right) \mathrm{Cz}_{q}\left(X_{\mathrm{cg}}\right)=\mathrm{Cz}_{\alpha} \mathrm{Cm}_{q}^{*} .
$$

The term on left side of the above equality will be defined as the Aerodynamic Invariant and consequently the term on the right side, too. The invariant aspect lies in the fact that the $\mathrm{Cm}_{q}^{*}$ term is independent of the center of gravity position as one can notice. This parameter only depends on the aerodynamic forces reduction points as the neutral point, the reduction point in pitch rate effect, and the "new" point obtained from the analytical identification of the pitch damping derivative (5). Moreover, this invariant only depends on Mach number for the rigid aircraft and on Mach number and the inertial characteristics for the flexible aircraft. Expression (11) is remarkable because whatever the center of gravity position, the product and the difference between the fundamental aerodynamics derivatives lead to a constant term. This constant term results from the product between the aircraft lift curve slope $\mathrm{Cz}_{\alpha}$ and the $\mathrm{Cm}_{q}^{*}$ term. Based on the aerodynamic invariant identification, it is possible now to provide an answer to the second question. 


\subsection{Answer to the Second Question Based on the Aerodynamic Invariant Contribution}

To answer the second question relative to the current manoeuvre point expression inconsistency addressed in subsection 2.1, let consider the definition of the manoeuvre point in steady pull-up defined as: "The manoeuvre point power-off corresponds to the specific position of the center of gravity cancelling the elevator deflection per $g$ numerator." The elevator deflection per $g$ function is $[4,5]$ :

$$
\Delta \delta q=-\frac{g L_{\mathrm{ref}}}{V^{2}} \frac{\mathrm{Cz}_{\alpha} \mathrm{Cm}_{q}+\mathrm{Cm}_{\alpha}\left(\mu-\mathrm{Cz}_{q}\right)}{\mathrm{Cz}_{\alpha} \mathrm{Cm}_{\delta q}-\mathrm{Cm}_{\alpha} \mathrm{Cz}_{\delta q}} \Delta n_{z} .
$$

The below relation constitutes the basic equation allowing to identify the analytical manoeuvre point expression. The numerator can be written by revealing explicitly the dependence of some aerodynamic derivatives to the center of gravity position, so:

$$
\mathrm{Cz}_{\alpha} \mathrm{Cm}_{q}\left(X_{\mathrm{cg}}\right)+\mathrm{Cm}_{\alpha}\left(X_{\mathrm{cg}}\right)\left(\mu-\mathrm{Cz}_{q}\left(X_{\mathrm{cg}}\right)\right)=0 .
$$

Now, by distributing the static stability derivative (3) in the right member, the following expression is obtained:

$$
\underbrace{\mathrm{Cz}_{\alpha} \mathrm{Cm}_{q}\left(X_{\mathrm{cg}}\right)-\mathrm{Cm}_{\alpha}\left(X_{\mathrm{cg}}\right) \mathrm{Cz}_{q}\left(X_{\mathrm{cg}}\right)}_{\text {Aerodynamic invariant }}+\mu \mathrm{Cm}_{\alpha}\left(X_{\mathrm{cg}}\right)=0
$$

where the aerodynamic invariant defined previously (11) can be identified. So, by replacing it, one obtains:

$$
\mathrm{Cz}_{\alpha} \mathrm{Cm}_{q}^{*}+\mu \mathrm{Cm}_{\alpha}\left(X_{\mathrm{cg}}\right)=0 .
$$

The only dependence of the above expression compared to the center of gravity position is ensured by the static stability derivative $\mathrm{Cm}_{\alpha}\left(X_{\mathrm{cg}}\right)(3)$. Finally, by substituting (3) in (12), the manoeuvre point expression power-off is defined as follows [5]:

$$
\frac{X_{M P}}{L_{\mathrm{ref}}}=\left(\frac{X_{F}}{L_{\mathrm{ref}}}\right)_{\alpha}-\left(\frac{\mathrm{Cm}_{q}^{*}}{\mu}\right) .
$$

Finally, with the above expression identification (13), it is possible to answer the second question addressed in subsection 2.1: "We can exhibit an analytical manoeuvre point expression independent of the center of gravity position." This expression is structured like the current one (2) [3]. Nevertheless, with a main difference, this one is unconditionally independent of the center of gravity position, due to the $\mathrm{Cm}_{q}^{*}$ term (9) contribution and so the aerodynamic invariant. Now, after having exhibited the aerodynamic invariant, the following subsection interprets the aerodynamic invariant meaning. 


\subsection{Interpretation of the Aerodynamic Invariant}

To interpret the aerodynamic invariant meaning, let consider the expression (8) which is for reference:

$$
\mathrm{Cm}_{q}\left(X_{\mathrm{cg}}\right)=-\mathrm{Cz}_{\alpha}\left(\frac{X_{\mathrm{cg}}}{L_{\mathrm{ref}}}-\left(\frac{X_{F}}{L_{\mathrm{ref}}}\right)_{\alpha}\right)\left(\frac{X_{\mathrm{cg}}}{L_{\mathrm{ref}}}-\left(\frac{X_{F}}{L_{\mathrm{ref}}}\right)_{q}\right)+\mathrm{Cm}_{q}^{*} .
$$

Now, by considering that the center of gravity coincides with the aircraft neutral point, which can be probable in an aircraft with a relaxed static stability, the expression (14) becomes:

$$
\mathrm{Cm}_{q}\left(X_{F, \alpha}\right)=\mathrm{Cm}_{q}^{*} .
$$

This result shows that the $\mathrm{Cm}_{q}^{*}$ term represents the pitch damping derivative at the neutral point. Consequently, the aerodynamic invariant defined previously (11) can be written as

$$
\mathrm{Cz}_{\alpha} \mathrm{Cm}_{q}\left(X_{\mathrm{cg}}\right)-\mathrm{Cm}_{\alpha}\left(X_{\mathrm{cg}}\right) \mathrm{Cz}_{q}\left(X_{\mathrm{cg}}\right)=\mathrm{Cz}_{\alpha} \mathrm{Cm}_{q}\left(X_{F, \alpha}\right) .
$$

This expression shows that the product and the difference between the fundamental aerodynamics derivatives lead to the aerodynamic invariant defined at the aircraft neutral point.

After the aerodynamic invariant interpretation, the following part proposes to widen the field of this study in order to check whether the aerodynamic invariant influence the aircraft dynamics characteristic parameters or not. One will limit the mode frequency to the short period, the damping ratio, and the stability limit point of the short period mode model.

\subsection{Effect of the Aerodynamic Invariant on the Aircraft Dynamics Parameters}

This part analyzes the natural aircraft dynamics characteristics parameters in order to detect the presence of the aerodynamic invariant. These parameters are the short period mode frequency, noted $\omega_{\mathrm{sp}}^{2}$, the damping ratio, noted $\xi_{\mathrm{sp}}$, and the dynamic stability limit point, noted $X_{\mathrm{DSL}} / L_{\text {ref }}$. In order to identify these parameters, let consider the linear short period mode model based on the lift and pitching moment equations in dimensional form [5]. Without going into calculation details, the short period mode frequency and the damping ratio are:

- square of the short period mode frequency $\omega_{\mathrm{sp}}^{2}$ :

$$
\omega_{\mathrm{sp}}^{2}=-\left(\frac{V}{L_{\mathrm{ref}}}\right)^{2} \frac{\mathrm{Cz}_{\alpha} \mathrm{Cm}_{q}+\mathrm{Cm}_{\alpha}\left(\mu-\mathrm{Cz}_{q}\right)}{\eta\left(\mathrm{Cz}_{\dot{\alpha}}+\mu\right)} ;
$$


- damping ratio $\xi_{\mathrm{sp}}$ :

$$
2 \xi_{\mathrm{sp}} \omega_{\mathrm{sp}}\left(\mathrm{Cz}_{\alpha}+\mu\right) \eta \frac{L_{\mathrm{ref}}}{V}=-\left(-\mathrm{Cz}_{\alpha} \eta+\mathrm{Cm}_{q}\left(\mathrm{Cz}_{\alpha}+\mu\right)+\mathrm{Cm}_{\alpha}\left(\mu-\mathrm{Cz}_{q}\right)\right) \text {. }
$$

Now, by substituting the pitch damping expression (10) in (15) and (16), one has the following relations:

- square of the short period mode frequency $\omega_{\mathrm{sp}}^{2}$ :

$$
\omega_{\mathrm{sp}}^{2}=-\left(\frac{V}{L_{\mathrm{ref}}}\right)^{2} \frac{\overline{{\mathrm{Cz_{ \alpha }} \mathrm{Cm}_{q}^{*}}}}{\eta\left(\mathrm{Cz}_{\dot{\alpha}}+\mu\right)}-\left(\frac{V}{L_{\mathrm{ref}}}\right)^{2} \frac{\mu \mathrm{Cm}_{\alpha}}{\eta\left(\mathrm{Cz_{ \alpha } + \mu )}\right.} ;
$$

- damping ratio $\xi_{\mathrm{sp}}$ :

$$
\begin{aligned}
& 2 \xi_{\mathrm{sp}} \omega_{\mathrm{sp}} \eta \frac{L_{\mathrm{ref}}}{V} \mathrm{Cz}_{\alpha}=-\left(\frac{1}{\mathrm{Cz}_{\alpha}+\mu}\right)\left(-\mathrm{Cz}_{\alpha}^{2} \eta+\mathrm{Cz}_{q} \mathrm{Cm}_{\alpha}\left(\mathrm{Cz}_{\alpha}+\mu\right)\right. \\
&\left.+\mathrm{Cz}_{\alpha} \mathrm{Cm}_{\alpha}\left(\mu-\mathrm{Cz}_{q}\right)\right)-\mathrm{Cz}_{\alpha} \mathrm{Cm}_{q}^{*} .
\end{aligned}
$$

The analysis of the two above expressions highlights the presence of the aerodynamic invariant and, therefore, its unquestionable influence. It is difficult to quantify its influence without calculations.

However, for conventional aircraft configurations with a negative $\mathrm{Cz}_{\alpha} \mathrm{Cm}_{q}^{*}$ value, when this one increases, that is, becomes less negative, then, for a given center of gravity position, the short period mode frequency and the damping ratio decrease. Consequently, it leads to a less damped aircraft. Furthermore, for the short period mode model, it can be demonstrated that the dynamic stability limit point, previously defined, coincides with the manoeuvre point position power-off given by the expression (13), so:

$$
\frac{X_{\mathrm{DSL}}}{L_{\mathrm{ref}}}=\left(\frac{X_{F}}{L_{\mathrm{ref}}}\right)_{\alpha}-\frac{\mathrm{Cm}_{q}^{*}}{\mu} .
$$

This result allows to notice, once again, the presence of the $\mathrm{Cm}_{q}^{*}$ term and, therefore, the aerodynamic invariant influence defined previously as $\mathrm{Cz}_{\alpha} \mathrm{Cm}_{q}^{*}$.

This part has highlighted the presence of the aerodynamic invariant $\mathrm{Cz}_{\alpha} \mathrm{Cm}_{q}^{*}$ on the aircraft dynamics characteristic parameters and, consequently, its unquestionable influence in the aircraft dynamics foundation. This statement can be enlarged to the natural aircraft transfer functions strongly linked to the aircraft dynamics characteristic parameters and so the aerodynamic invariant. However, after these developments, a question is addressed: "Which link is there between the Aerodynamic Invariant and New Aircraft Configurations?" An explanation is suggested through the discussion in the following subsection. 


\subsection{Aerodynamic Invariant and New Aircraft Configurations}

Beyond the predictive aspect is the improvement of some Handling Qualities parameters as the manoeuvre point position via the aerodynamic invariant contribution. The main question is up to what point this parameter disseminated in aircraft dynamics could be "challenged" in order to "seek" New Aircraft Configurations in conceptual design phase with improved Handling Qualities compared to the discussed current conventional configurations. More precisely, in the civil aircraft design process, the traditional geometrical form of type wing/fuselage/tailplane is frozen in the conceptual design phase. That means somewhere one "undergoes" this invariant calculated after the design phase. Now, a question is: "Would it be possible to "challenge" earlier this aerodynamic invariant in the conceptual design phase via the aircraft geometry in order to confer to the aircraft specific properties in terms of static and dynamic stability?" For example, through the manoeuvre point position, the short period mode frequency and damping ratio values are based on the natural or the closed loop aircraft. This high level questioning seems to be legitimate and comprehensible; however, the Aerodynamic Invariant Challenge means that it remains the vague that must be clarified.

For that, let have a look at the aerodynamic invariant expression (11), which is for reference:

$$
\mathrm{Cz}_{\alpha} \mathrm{Cm}_{q}^{*}=-\mathrm{Cz}_{\alpha}^{2}\left(\left(\frac{X_{F}}{L_{\mathrm{ref}}}\right)_{\alpha, q}^{2}-\left(\frac{X_{F}}{L_{\mathrm{ref}}}\right)_{\alpha}\left(\frac{X_{F}}{L_{\mathrm{ref}}}\right)_{q}\right) .
$$

The main idea behind the "Aerodynamic Invariant Challenge" concept consists in trying to reorganize the aerodynamic forces reduction points in the above expression by tuning the aircraft geometry in order to confer to this one increased Handling Qualities via the term $\mathrm{Cz}_{\alpha} \mathrm{Cm}_{q}^{*}$. For example, the main consequence of the $\mathrm{Cz}_{\alpha} \mathrm{Cm}_{q}^{*}$ reduction is the relax of the aircraft dynamics stability.

Furthermore, one of the important aspects of this approach consists in trying to "optimize" Handling Qualities by one or several criteria. Now, the question is what one understands by "optimized" Handling Qualities? One can bring a possible answer: "When one moves the dynamic system of its balanced position, namely, the aircraft, this one must be able to return to its initial or ordered position with less possible energy." For example, by simulating a commanded load factor, if the aircraft is able to reach this command with minimum control surface deflection in the frame of the civil aircraft requirements.

These questions had the aim to initiate a thought and to trace some possible prospects linked to the identification for the Aerodynamic Invariant Concept. After this rather theoretical section, the next section illustrates these considerations with numerical results. 


\section{AERODYNAMIC INVARIANT EFFECT ON AIRCRAFT DYNAMICS}

To illustrate the aerodynamic invariant effect on the aircraft dynamics, this part proposes to visualize the aircraft dynamic response in closed loop by varying the aerodynamic invariant for a manoeuvrability criterion. Subsections 3.13.3 present the data used to perform the calculation and the regulation loop characteristics. Subsection 3.4 presents the elevator time responses for a positive commanded load factor and for two values of the aerodynamic invariant. In order to approach this complex problem in a simple way, the numerical application has been considered with the short period mode model.

\subsection{Flight Point, Geometrical and Mass Characteristics}

Flight point: $\mathrm{M}=0.25 ; V_{\mathrm{tas}}=165 \mathrm{~m} / \mathrm{s}$; and $H=0$.

Geometrical and mass characteristics: $M=350 \mathrm{t} ; I_{y y}=2.46310 \cdot 10^{7} \mathrm{~kg} \cdot \mathrm{m}^{2}$; $S_{\text {ref }}=1243.4 \mathrm{~m}^{2} ; L_{\mathrm{ref}}=25.74 \mathrm{~m}$; and $L_{25 \%}=20.133 \mathrm{~m}$.

\subsection{Aerodynamic Flying Wing Characteristics}

In this subsection, the flying wing aerodynamics characteristics are provided through the natural short period mode model:

- neutral point position: $X_{F}^{\alpha}=37.9(\%) \mathrm{mac}$;

- manoeuvre point position power-off: $X_{P M}=39.51(\%) \mathrm{mac}$; and

- aerodynamic invariant value: $\mathrm{Cz}_{\alpha} \mathrm{Cm}_{q}^{*}=\mathrm{Cz}_{\alpha}(-0.29)$.

After providing the natural aircraft characteristics values, the next subsection proposes a sensitivity study of the aircraft dynamics response compared to the aerodynamic invariant in order to illustrate the so-called "Aerodynamic Invariant Challenge" (see subsection 2.7) based on a single short period mode model. This approach could be somewhere criticizable because by changing the value of the aerodynamic invariant, one modifies somehow the aircraft geometry. However, at this step of the study, it appears to be the only relevant mean to estimate the aerodynamic invariant impact on the aircraft dynamics response.

\subsection{Closed Loop Architecture}

To illustrate this manoeuvre criterion, the $C^{*}$ law is used commonly in control laws architecture in civil aircraft in order to increase the short period mode frequency and the damping ratio (Fig. 1). 


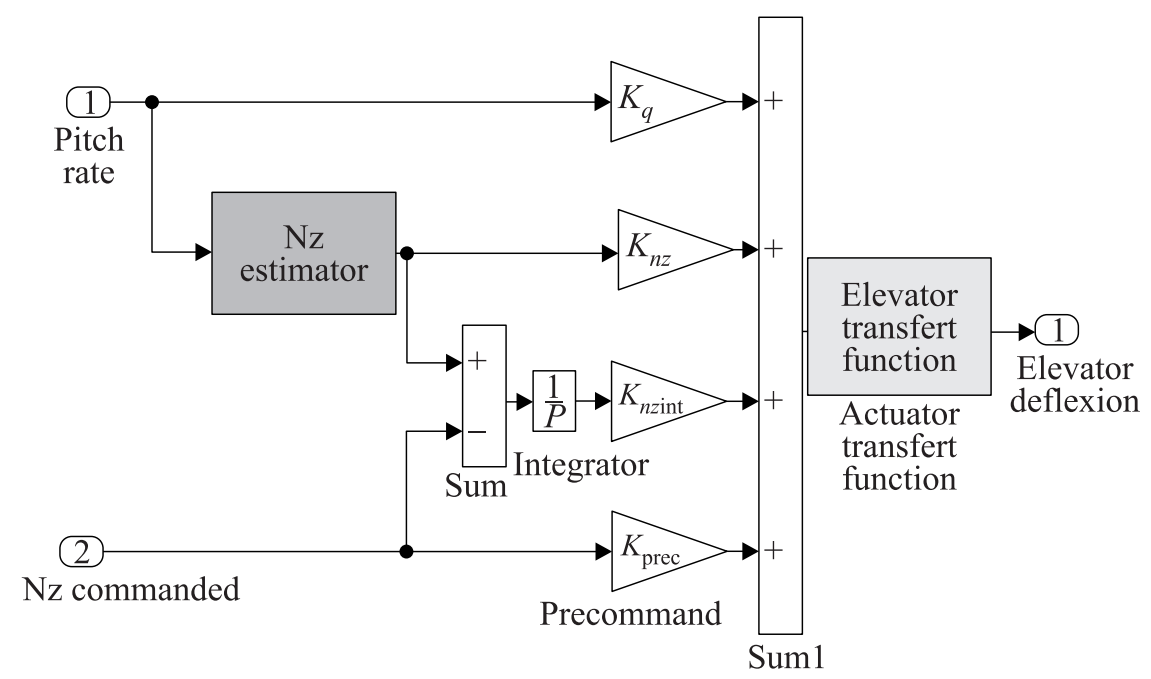

Figure 1 Architecture of the $C^{*}$ law

Actually, the gains, $K_{q}, K_{n z}$, and $K_{n z \text { int }}$ are calculated by targeting the short period mode frequency in closed loop for $\omega_{\mathrm{sp}}=2.2 \mathrm{rad} / \mathrm{s}$ and the damping ratio for $\xi_{\mathrm{sp}}=0.8$. This set of gains is calculated for each value of the aerodynamic invariant. As it is mentioned in the following subsection, one value of the aerodynamic invariant is associated with a "nominal case" and another value is associated with a "challenged case." To achieve the manoeuvre criterion calculation, an input as a commanded load factor in a crenel form with a maximum value equal to $n_{z c}=0.5 \mathrm{~g}$ is considered. In the following subsection, the time simulation results are presented.

\subsection{Results}

This subsection presents the elevator time responses for a center of gravity position at $X_{\mathrm{cg}}=39 \%$ and for two values of the aerodynamic invariant via the $\mathrm{Cm}_{q}^{*}$ term. The "nominal case" is considered for $\mathrm{Cm}_{q}^{*}=-0.29$ and the "challenged case" is considered for $\mathrm{Cm}_{q}^{*}=0$.

Figure 2 shows an important result: the aerodynamic invariant cancellation allowed to reduce the elevator deflection amplitude. In other words, the commanded load factor for the challenged case requires less control surface deflection to nose up compared to the nominal case. For this center of gravity position, a 26 percent reduction of the control surface activity to nose up is observed. This property revealed by the simulation, confirmed for any center of gravity position, constitutes an important point. 


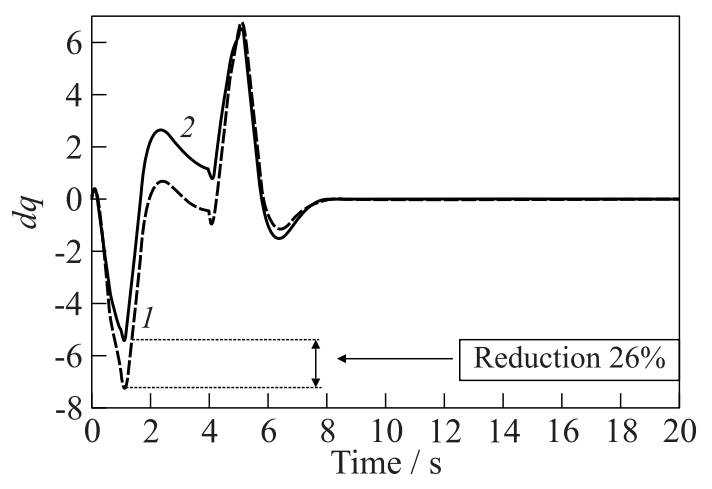

Figure 2 Elevator deflection for "nominal case," $\mathrm{Cm}_{q}^{*}=-0.29(1)$ and for "challenged case," $\mathrm{Cm}_{q}^{*}=0(2)$

This interesting result can be explained in a simple way by considering the pitch damping derivative formulated according to expression (10). It shows that the cancelation of the $\mathrm{Cm}_{q}^{*}$ term induces a lower pitch damping derivative. This result means less dynamic stability, hence, more manoeuvrability, and, consequently, less control surface deflection to nose up. It can be concluded that the $\mathrm{Cm}_{q}^{*}$ term seems to be a relevant parameter in order to have an aircraft with a relaxed dynamic stability but with an increased manoeuvrability. Furthermore, a reduction of the elevator deflexion to nose down is observed for a negative commanded load factor.

At last, Fig. 2 shows that the maximum value of the control surface deflection remains almost insensitive with the reduction of the aerodynamic invariant.

\section{CONCLUDING REMARKS}

This paper highlights the Aerodynamic Invariant Concept and traces the prospects in order to identify a possible way to "seek" New Aircraft Configurations with improved Handling Qualities compared to the current aircraft configurations. The Aerodynamic Invariant allowed to identify an accuracy form of the manoeuvre point expression power-off [5].

Based on a manoeuvrability criterion in closed loop, the major result highlights a reduction of the noseup (nosedown) control surface deflection with a decrease of the Aerodynamic Invariant $\mathrm{Cz}_{\alpha} \mathrm{Cm}_{q}^{*}$. This result can allow a drag reduction while ensuring optimized Handling Qualities as previously defined in subsection 2.7. The aerodynamic invariant seems to be a relevant parameter in order to "seek" New Aircraft Configurations with improved Handling Qualities via the geometry. 
Future works should consist in extending this approach to others Handling Qualities criteria in the whole flight domain. It could be extended by improving the aircraft model, for example, by introducing the drag equation, the various power-plants, turbofan or C.R.O.R (Contra Rotative Open Rotor), and the aerodynamics nonlinearities. To conclude, the fundamental question whose answer could inspire us, is: "What about this Aerodynamic Invariant for birds?"

\section{REFERENCES}

1. Rodden, W. P., J. P. Giesing, and T. P. Kalman. 1970. New development and application of the subsonic doublet-lattice method for nonplanar configurations. AGARD Symposium, Unsteady Aerodynamics for Aeroelastic Analyses of Interfering Surfaces. Tønsberg Oslofjorden. AGARD Paper No. 4.

2. http://www.dept.aoe.vt.edu/ mason/Mason_f/CAtxtChap6.pdf.

3. Roskam, J. 1979. Airplane flight dynamics and automatic flight controls. Part I: Rigid aircraft flight dynamics (open loop). 1st ed. Roskam Aviation and Engineering Corp.

4. De Castro, H. V. 2001. The longitudinal static stability of tailless aircraft. COA Report No. 0018/1.

5. Bazile, J. 2007. Proposal of a new analytic formulation of the manoeuvre point in steady manoeuvre. EUCASS. Brussels. 